\title{
Basaltos de San Pablo: un bloque de un arco de islas en el norte de la cordillera Central de Colombia. Caracterización petrográfica y química
}

\author{
Gabriel Rodríguez-García $^{{ }^{*}}$; Carlos Mario Celada-Arango ${ }^{1}$ \\ DOI: http://dx.doi.org/10.18273/revbol.v40n2-2018004 @ (1) \\ Forma de citar: Rodríguez-García, G., y Celada-Arango, C.M. (2018). Basaltos de San Pablo: un bloque de un \\ arco de islas en el norte de la cordillera Central de Colombia. Caracterización petrográfica y química. Boletín de \\ Geología, 40(2), 69-85. DOI: 10.18273/revbol.v40n2-2018004.
}

\begin{abstract}
RESUMEN
Los Basaltos de San Pablo son un bloque de rocas volcánicas que afloran junto a sedimentitas marinas, localizadas en el norte de la Cordillera Central de Colombia, entre bloques de rocas metamórficas del Complejo Cajamarca e intruidos por el Batolito Antioqueño de edad Cretácica superior. Los basaltos de San Pablo están constituidos por basaltos (diabasas espilitizadas), con texturas intergranular a ofítica-subofítica y ocasionalmente porfídica y variolítica - subofítica, similares a las rocas de corteza oceánica del Complejo Quebradagrande y las Diabasas de San José de Urama. Los resultados de óxidos de elementos mayores sugieren que corresponden a basaltos y andesitas basálticas calcoalcalinas, con contenidos de $\mathrm{SiO}_{2}$ entre 43,13\% y 55,36\%, con concentraciones de $\mathrm{MgO}$ en el rango entre 5,85\% y 9,45\% y valores de $\mathrm{TiO}_{2}$ entre $1 \%$ y $2 \%$. Los diagramas multielementales de tierras raras y elementos trazas muestran anomalías negativas de $\mathrm{Nb}$ y positivas de $\mathrm{Cs}, \mathrm{Ba}, \mathrm{K}$ y $\mathrm{Pb}$, que sugieren un ambiente de arco, con un patrón de REE similar al de basaltos de corteza oceánica de tipo MORB en las rocas menos diferenciadas, pero con valores mayores de Th que los ubican dentro de un ambiente de arco de islas.
\end{abstract}

Palabras clave: Cordillera Central; Geoquímica; Petrografía; Andes del norte.

\section{San Pablo Basalts: an island arc block in the north of the Central Cordillera of Colombia. Petrographic and chemical characterization}

\begin{abstract}
The San Pablo Basalts constitute an exotic block of volcanic rocks that crop out next to marine sediments, located in the Northern part of Colombia's Central Cordillera, between metamorphic blocks of the Cajamarca Complex and intruded by the Antioquia Batholith, Upper Cretaceous in age. The San Pablo Basalts are composed of basalts (spilitic diabases), with intergranular to phiticsubophitic and occasionally porphyritic and variolitic-subophitic textures, similar to the oceanic crust rocks of the Quebradagrande Complex and the San Jose de Urama Diabases. The higher oxides results suggest that the rocks correspond to calc-alkaline, basalts and basaltic andesites, with $\mathrm{SiO}_{2}$ contents between $43.13 \%$ and $55.36 \%$, MgO concentration ranges between $5.85 \%$ and $9.45 \%$ and $\mathrm{TiO}_{2}$ values between $1 \%$ and $2 \%$. Multi-element diagrams of rare earth elements and trace elements show a negative $\mathrm{Nb}$ anomaly and positive anomalies of $\mathrm{Cs}, \mathrm{Ba}, \mathrm{K}$ and $\mathrm{Pb}$, which suggests an arc environment with an REE trend similar to MORB-type oceanic crust basalts in the less differentiated rocks, but with higher Th values, which relates them to an island arc environment.
\end{abstract}

Keywords: Central Cordillera; Geochemistry; Petrography; Northern Andes.

\footnotetext{
${ }^{1}$ Grupo de Estudios Geológicos Especiales, Servicio Geológico Colombiano, Medellín, Colombia. (*) grodriguez@sgc.gov.co; cmcelada@sgc.gov.co
} 


\section{INTRODUCCIÓN}

Los Basaltos de San Pablo, fueron descritos por Hall et al. (1972) bajo el nombre de Metabasaltos del Cretácico inferior; se encuentran intercalados con sedimentitas de la Formación San Pablo, siendo considerados contemporáneos. Posteriormente González (2001) los denominó Metabasaltos de San Pablo.

La unidad Basaltos de San Pablo abarca un área aproximada de $135 \mathrm{~km}^{2}$, corresponde a un cuerpo de forma alargada en dirección Norte - Sur que aflora en los municipios de Guadalupe y Campamento, en el departamento de Antioquia, sobre el eje de la Cordillera Central de Colombia; en el sector occidental se encuentra intruida por lentes de metagabros, la parte sur se encuentra truncada e intruida por rocas del Batolito Antioqueño de edad Cretácica superior (Hall et al., 1972); al norte presenta contactos fallados con las metamorfitas de la Cordillera Central de posible edad triásica y hacia la parte superior la secuencia se intercala con sedimentitas de la Formación San Pablo sin edad definida, pero que han sido consideradas del Cretácico inferior por correlación con el Complejo Quebradagrande (Hall et al., 1972).

Las rocas que conforman la unidad fueron descritas como flujos de basaltos espilíticos con texturas ofíticas a subofiticas, posiblemente submarinos y brechas de flujos (Hall et al., 1972; González, 2001).

La unidad Basaltos de San Pablo se presenta como un bloque independiente que aflora en el norte de la Cordillera Central de Colombia entre las fallas Otú - Pericos y Cauca-Almaguer, dentro del basamento metamórfico de la Cordillera Central, el cual está constituido por paraesquistos y paraneises, cuarcitas, mármoles, anfibolitas, esquistos verdes y granitos de tipo S (Echeverría, 1973; Restrepo y Toussaint, 1984; Maya 2001), que representan bloques metamórficos de edades paleozoicas, pérmicas, triásicas, jurásicas y cretácicas, cuyos límites no han sido establecidos dentro de la cartografía del norte de la Cordillera Central de Colombia. La unidad Basaltos de San Pablo está ubicada a unos $80 \mathrm{~km}$ al Este de la zona de sutura con el basamento oceánico de la Cordillera Occidental, que se localiza al oeste de la Falla Cauca-Almaguer (Restrepo y Toussaint, 1988; Maya y González, 1995; Nivia et al., 1996; Kerr et al., 1997) (FIGURA 1).

Dicho basamento está constituido por basaltos (diabasas) de afinidad T-MORB, junto con andesitas, plutones tonalíticos y dioríticos de arco con afinidad toleítica a calcoalcalina media en K (Rodríguez et al., 2012; Rodríguez y Arango, 2013; Rodríguez y Zapata, 2013).

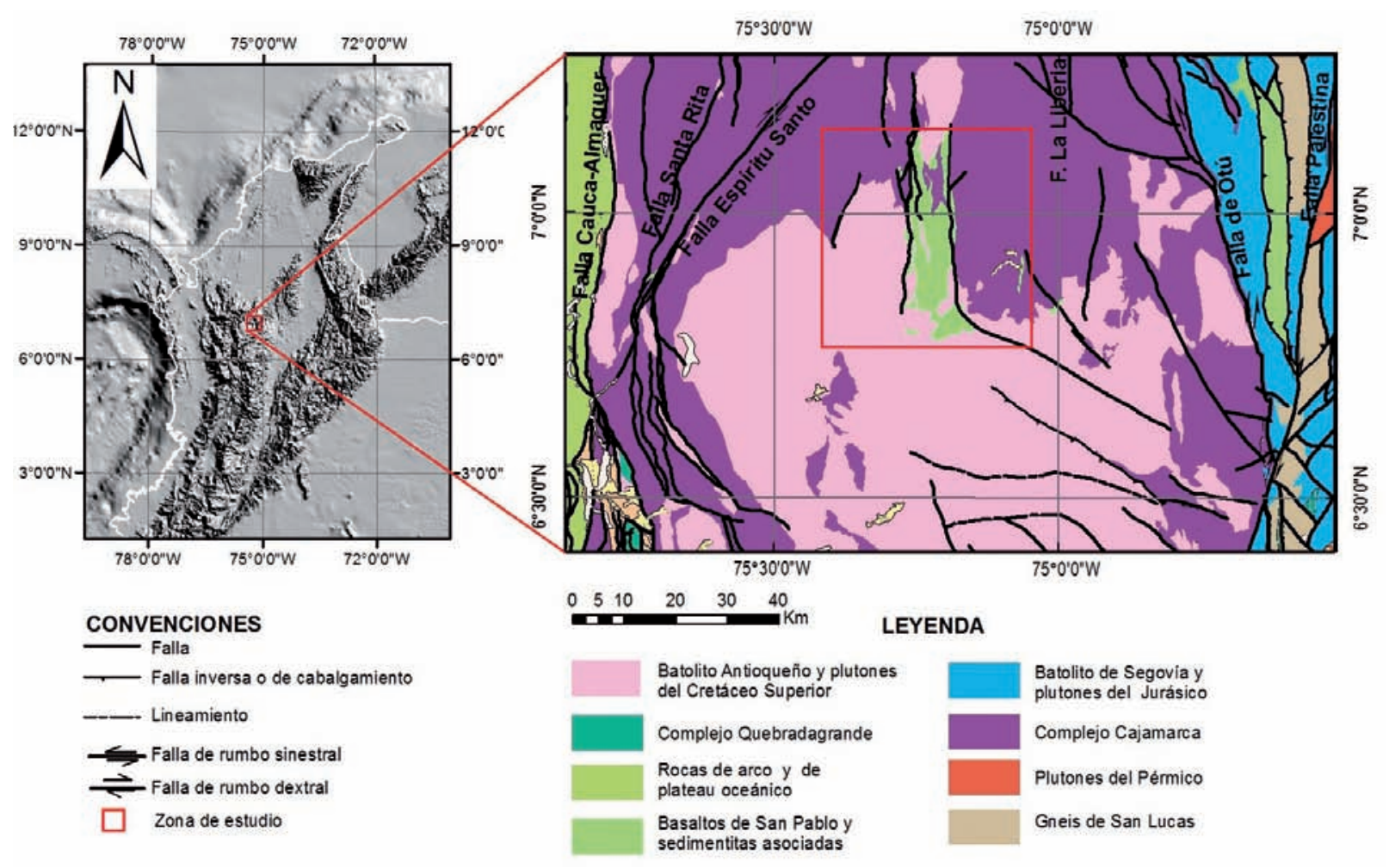

FIGURA 1. Geología Regional alrededor de los Basaltos de San Pablo (modificada de González, 2001). 
Mediante el análisis de nueva información geoquímica y la revisión petrográfica de las secciones delgadas de rocas de la unidad Basaltos de San Pablo, se caracteriza litológicamente la unidad y se compara con datos litogeoquímicos y petrográficos de basaltos (diabasas) asociadas al Complejo Quebradagrande (Rodríguez y
Cetina, 2016) y con datos publicados por Rodríguez y Arango (2013) para rocas de la unidad Diabasas de San José de Urama, que se correlacionan con el Grupo Diabásico al sur de la Cordillera Occidental en Colombia (FIGURA 2).

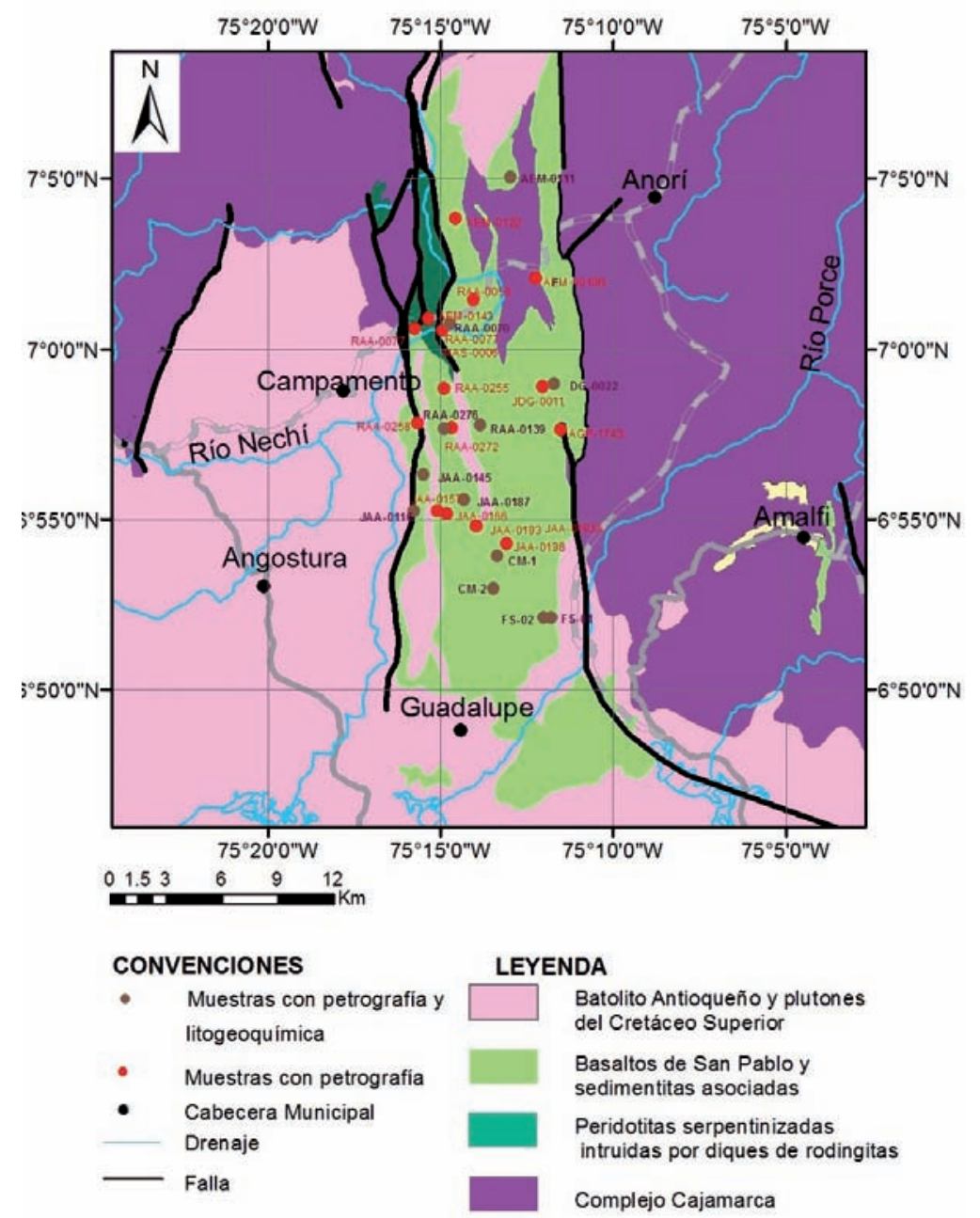

FIGURA 2. Localización y muestreo de afloramientos de los Basaltos de San Pablo.

\section{MATERIALES Y MÉTODOS}

Se realizó una revisión petrográfica de 25 secciones delgadas colectadas durante la época del Inventario Minero Nacional en los trabajos regionales de cartografía geológica del cuadrángulo H8. Se separaron las rocas con texturas ofíticas, subofíticas, intergranulares, intersectales y variolíticas, menos afectadas por alteraciones y deformaciones dinámicas, se determinó su distribución espacial dentro de la unidad de los Basaltos de San Pablo. Se tomaron 4 muestras nuevas en el terreno para completar un total de 29 secciones delgadas de las rocas de la unidad.
Doce muestras de roca fueron analizadas químicamente, 9 de estas en el laboratorio del Servicio Geológico Colombiano (SGC) y 3 en el laboratorio de la Universidad de Cardiff - Inglaterra. Las muestras fueron seleccionadas considerando el menor grado de alteración y deformación, ya que la unidad se encuentra en el contacto entre las rocas metamórficas y las del Batolito Antioqueño, presentando comúnmente alteraciones deutéricas. Los contenidos de óxidos de elementos mayores y de los elementos trazas $\mathrm{V}$, Mo, $\mathrm{Nb}$, Ta, W, Zr y Hf fueron determinados con el método de fluorescencia de rayos X para las muestras analizadas en el SGC. Para el resto de elementos traza y tierras raras 
se utilizó el equipo de espectrometría de masas con plasma acoplado inductivamente (ICP-MS). Para las tres muestras analizadas en la Universidad de Cardiff (INM-5271, INM-5324, INM-6180) se utilizó el método ICP-MS, tanto para los óxidos de elementos mayores como para los elementos trazas. En la interpretación de los resultados geoquímicos las concentraciones de los óxidos de los elementos mayores fueron recalculados en base seca, teniendo en cuenta los valores de LOI (perdidas por ignición).

Los resultados químicos de los análisis de las rocas, se compararon con los datos geoquímicos publicados para las rocas de la unidad Diabasas de San José de Urama (Rodríguez y Arango, 2013), así como con los basaltos (diabasas) de corteza oceánica del Complejo Quebradagrande (Rodríguez y Cetina, 2016), con el objeto de determinar si ambos grupos de rocas muestran comportamientos geoquímicos similares y si corresponden a basaltos generados en ambientes geotectónicos similares.

La palabra diabasa se utiliza en este manuscrito en el sentido textural para rocas de composición basáltica que tienen texturas ofitica, subofítica, intergranular e intersectal. El énfasis en el nombre textural "diabasa” a lo largo del texto, se debe a que en el noroccidente de los Andes de Colombia las unidades con basaltos de corteza oceánica como el Grupo Diabásico, las Diabasas de San José de Urama y los basaltos (diabasas) de corteza oceánica de tipo N_MORB del Complejo Quebradagrande, presentan características texturales de "diabasas" formadas a partir de derrames lávicos y no de diques. Algunas rocas basálticas que conforman la Unidad Basaltos de San Pablo, presentan rasgos texturales similares al de rocas de corteza oceánica de las unidades antes mencionadas.

\section{RESULTADOS}

\section{Características petrográficas de los Basaltos de San Pablo}

La unidad Basaltos de San Pablo está constituida predominantemente por basaltos y ocasionales gabros; localmente se presentan brechas volcánicas. Las rocas han sido afectadas hacia los contactos con las rocas metamórficas por deformación frágil y dúctil, presentando localmente metamorfismo dinámico y estructura foliada (milonitas y protomilonitas) y rocas con deformación frágil, con trituración parcial y completa de los minerales (cataclasitas). Por otra parte, la unidad fue afectada en sus bordes por alteración deutérica de piroxenos a anfíboles con hábito fibroso, introducción de venillas de epidota y alteración de plagioclasa y piroxeno a epidota, sericita y calcita (alteración propilítica). Predominan los basaltos con texturas ofítica, subofítica, intergranular, intersectal, variolítica y ofítica-porfídica con escasos fenocristales de plagioclasa; en algunos casos se presentan amígdulas rellenas de clorita, calcita y cuarzo (FIGURA 3).

Los minerales constituyentes son plagioclasa (generalmente poco alterada) y clinopiroxeno (frecuentemente uralitizado a anfíbol fibroso); los minerales accesorios corresponden a titanita y opacos; las alteraciones son a saussurita, sericita en la plagioclasa, uralita y carbonatos a partir del piroxeno y leucoxeno a partir de titanita. Como minerales de introducción se reconocieron epidota, clorita, mica blanca y cuarzo, rellenando fracturas y amígdulas o reemplazando al piroxeno y a la plagioclasa (TABLA 1).

El contenido de plagioclasa varía entre $45 \%$ y 55\%; ésta se presenta en cristales euhedrales a subhedrales de habito tabular alargado, entrecruzados y con clinopiroxeno intergranular frecuentemente uralitizado (el anfíbol puede ser fibroso), en las rocas basálticas, los bordes de los cristales varían de irregulares a rectos, con desarrollo de maclas de tipo Carlsbad y albita - Carlsbad mal desarrolladas, frescos o alterados a agregados secundarios de sericita, saussurita y clorita. Pueden presentarse como inclusiones poiquilíticas en clinopiroxeno con desarrollo de textura ofítica. En basaltos microcristalinos predominan los cristales tabulares con formas subhedrales formando ramilletes variolíticos de cristales junto a vidrio y piroxeno.

El piroxeno (augita?) se encuentra como cristales anhedrales a subhedrales de tamaño promedio entre 0,1 $\mathrm{mm}$ y $1,0 \mathrm{~mm}$, intergranulares con plagioclasa y formando texturas subofíticas. El piroxeno presenta clivaje moderadamente desarrollado y fracturas irregulares, relieve alto, son incoloros a ligeramente pardos con extinción ondulatoria, el color de birrefringencia máximo varía entre el amarillo del primer orden al rojo del segundo orden, con frecuencia se encuentra reemplazados por anfíbol (uralitización) de color verde pálido con hábito fibroso y ocasionalmente por calcita, pueden estar localmente molidos por deformación frágil; el ángulo de extinción es de alrededor de $41^{\circ}$.

Las amígdulas por lo general son de forma esférica con tamaños entre $0,5 \mathrm{~mm}$ y $3 \mathrm{~mm}$, rellenas por agregados complejos micro laminares de clorita de color verde pálido y birrefringencia azul Berlín, puede aparecer calcita en agregados, feldespato radial y cuarzo. 


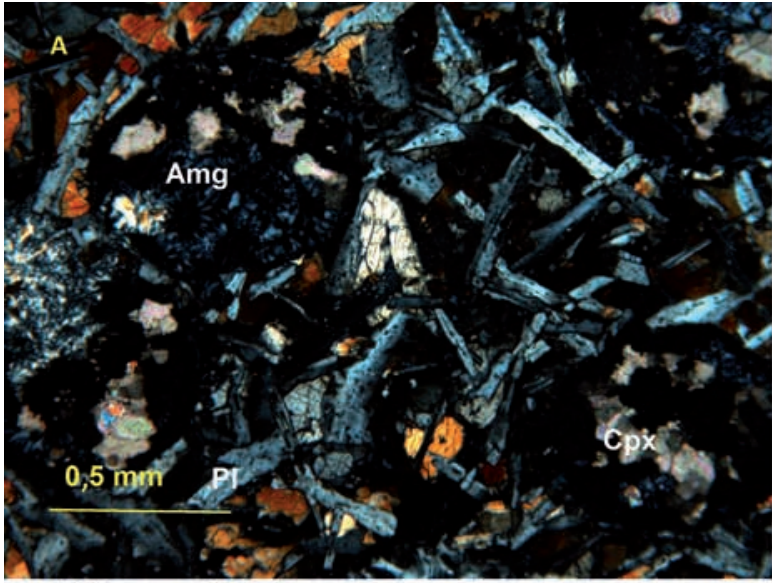

INM- 5367 - Basalto

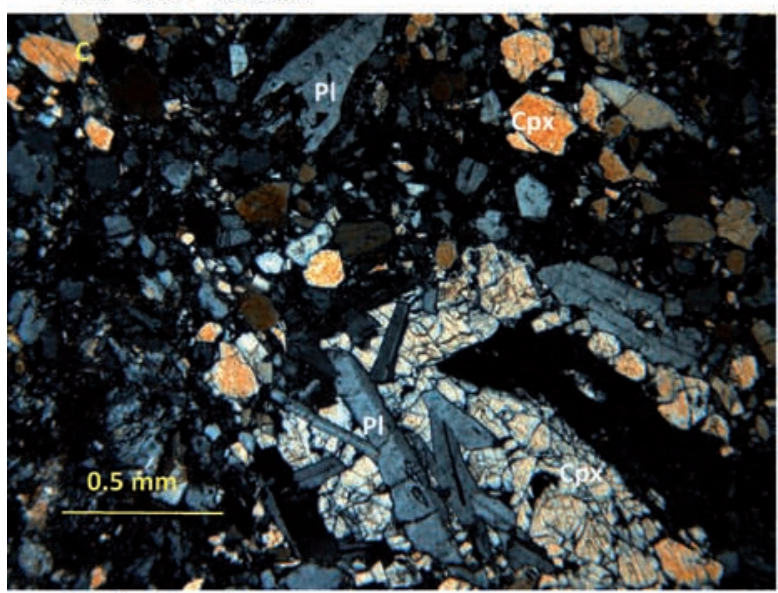

CM-1 - Basalto

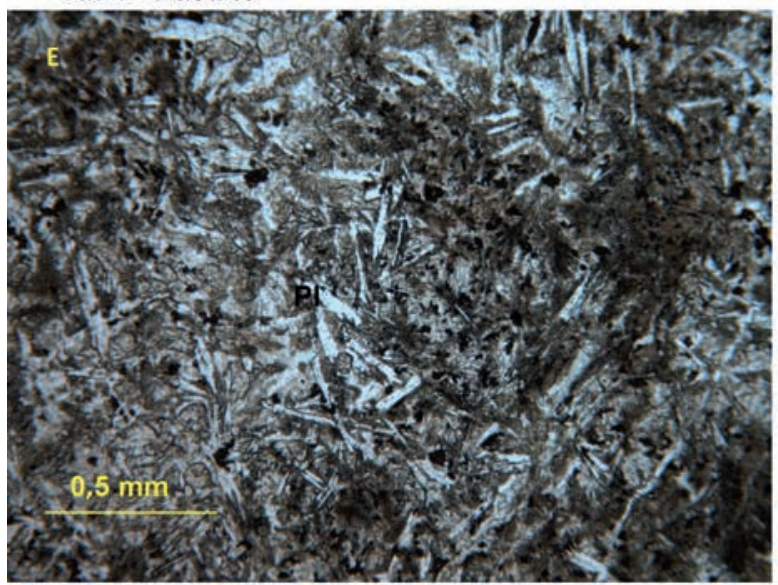

INM-5171 - Basalto

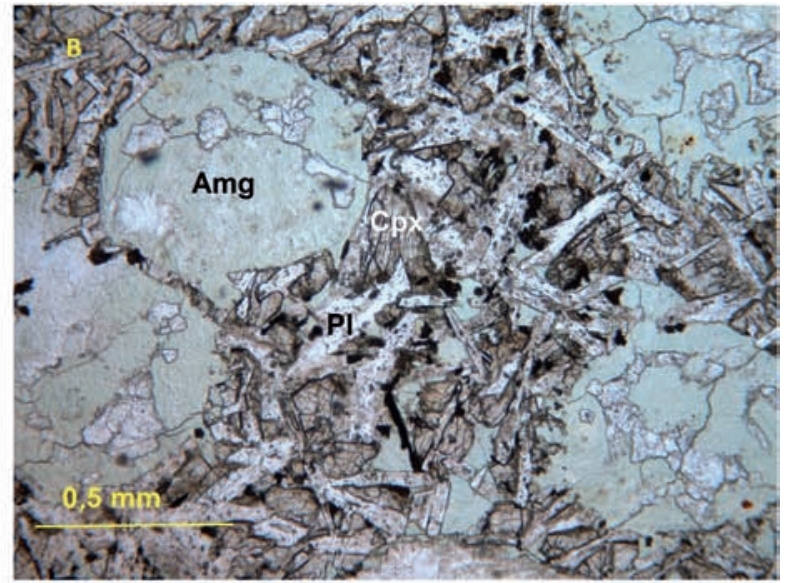

INM- 5367 - Basalto

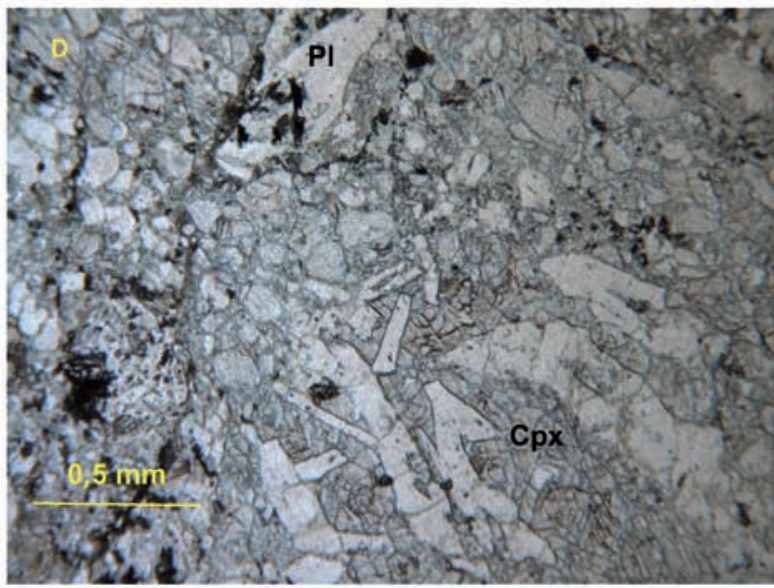

CM-1 - Basalto

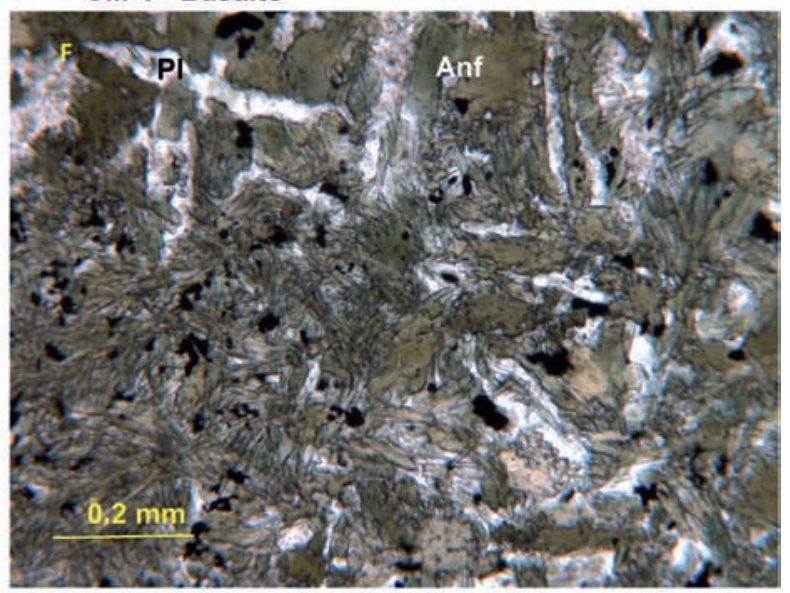

INM-5171 - Basalto

FIGURA 3. Características composicionales y texturales de las rocas de la unidad Basaltos de San Pablo: A. y B. Textura intergranular amigdular (PPL, XPL, 10x), C. y D. Textura subofítica con micro fallas (PPL, XPL, 10x) E. Textura variolítica (PPL, 10x). F. Textura intersectal con anfíbol uralítico a partir de piroxeno. Símbolos: Pl: plagioclasa, Cpx: piroxeno, Anf: anfíbol y Amg: amígdula. 
Basaltos de San Pablo: un bloque de un arco de islas en el norte de la cordillera Central de Colombia. Caracterización petrográfica y química

TABLA 1. Composición modal de los Basaltos de San Pablo.

\begin{tabular}{|c|c|c|c|c|c|c|c|c|c|c|}
\hline INM & Estación & C. Este & C. Norte & Pl & Cpx & Anf & Op & Ttn & otros & Clasificación \\
\hline 5015 & MAS-0006 & 870540 & 1266980 & 71 & 25 & & 4 & & $\operatorname{tr}$ & Basalto \\
\hline 5054B & AEM-0010B & 875520 & 1269800 & 53 & & 46 & 1 & & & Basalto \\
\hline 5119 & RAA-0058 & 872220 & 1268660 & 42 & & 57 & 1 & & & Basalto \\
\hline 5185 & RAA-0070 & 870940 & 1267320 & 83 & 9 & 2 & 2 & & 4 & Basalto \\
\hline 5187 & RAA-0072 & 870940 & 1267320 & 52,3 & & 44,2 & 2,5 & & 1 & Basalto \\
\hline 5192 & RAA-0077 & 869080 & 1267080 & 43,7 & & 50,6 & 1,7 & & 4 & Basalto \\
\hline 5271 & RAA-0139 & 872560 & 1261860 & 48,9 & 48,8 & & & 2,3 & & Basalto \\
\hline 5309 & RAA-0258 & 869180 & 1262000 & 73,8 & 20,7 & 3 & 1 & & 2,4 & Micro gabro \\
\hline 5311 & RAA-0255 & 870610 & 1263875 & 58,4 & 15,3 & 24,7 & 1,6 & TR & & Basalto \\
\hline 5312 & RAA-0276 & 870640 & 1261700 & 58,7 & 38,9 & & 1,6 & 0,8 & $\mathrm{TR}$ & Basalto \\
\hline 5324 & JAA-0116 & 869000 & 1257240 & 63,2 & 34 & & 2,5 & & 3 & Basalto \\
\hline 5331 & JAA-0157 & 870240 & 1257260 & 70,7 & 27 & 1,3 & 1 & $\mathrm{TR}$ & & Basalto \\
\hline 5334 & JAA-0166 & 870740 & 1257100 & 70,9 & 26,3 & & 0,7 & 1,1 & 1 & Basalto \\
\hline 5365 & JAA-0187 & 871660 & 1257860 & 37,3 & 59,2 & & & 3,1 & 0,4 & Basalto \\
\hline 5366 & JAA-0193 & 872300 & 1256400 & 50,1 & 42,1 & & TR & 1,2 & 6,6 & Basalto \\
\hline 5367 & JAA-0193A & 872300 & 1256400 & 52,6 & 38,5 & & & 1,1 & 7,8 & Basalto \\
\hline 5368 & JAA-0198 & 873940 & 1255460 & 61,5 & 37,6 & & 0,5 & 0,4 & 1,3 & Basalto \\
\hline 6176 & AGP-1743 & 876850 & 1261625 & 63 & & 24 & $\operatorname{tr}$ & & 13 & Milonita de Basalto \\
\hline 6178 & JDG-0011 & 875860 & 1263960 & 40 & & 59 & & 1 & & Basalto \\
\hline 6180 & JDG-0022 & 876480 & 1264100 & 37 & & 22 & & 1 & 40 & Basalto \\
\hline 6772 & AEM-0111 & 874170 & 1275280 & 55 & & 35 & 1 & 1 & 5 & Basalto \\
\hline 6774 & AEM-0122 & 871270 & 1273060 & 44 & & 50 & 1 & 1 & 4 & Basalto \\
\hline 6777 & AEM-0143 & 869760 & 1267640 & 54 & & 25 & 1 & 1 & 19 & Basalto \\
\hline 6811 & RAA-0272 & 871040 & 1261720 & 65 & 25 & & 1 & & 10 & Gabro sausuritico \\
\hline \multirow[t]{3}{*}{6816} & JAA-0145 & 869520 & 1259220 & 60 & 35 & & 1,5 & & 5,5 & Micro gabro \\
\hline & CM-1 & 873449 & 1254792 & 48,8 & 49,7 & & 1,1 & 0,4 & & Basalto \\
\hline & CM-2 & 873236 & 1253055 & 48 & 46,3 & & & 4,3 & 1 & Basalto \\
\hline 900855 & FS-01 & 876319 & 1251464 & 23 & & 75,8 & 0,4 & 0,4 & & Basalto \\
\hline
\end{tabular}

\section{Geoquímica}

En la TABLA 2 se presentan los resultados de las concentraciones de los óxidos de elementos mayores de las rocas basálticas de la unidad Basaltos de San Pablo, las cuales presentan valores de $\mathrm{SiO}_{2}$ entre 43,13\% y 55,36\%, concentraciones de $\mathrm{MgO}$ en el rango entre 5,85 y 9,45\%, $\mathrm{Fe}_{2} \mathrm{O}_{3}$ que varía entre 8,22 y $11,42 \%$, contenidos de $\mathrm{Al}_{2} \mathrm{O}_{3}$ entre 14 y $17,6 \%$, valores $\mathrm{CaO}$ entre $7,16 \%$ y $12,53 \%$, la concentración de $\mathrm{TiO}_{2}$ en la mayoría de las muestras varía entre 1 y $2 \%$ (más altos que en las rocas de las Diabasas de San José de Urama que presenta valores $<1 \%$ y similares a los basaltos (diabasas) del Complejo Quebradagrande). Las muestras (FS-01 y FS-02) presentan valores mayores a 3\% en $\mathrm{TiO}_{2}$ y la muestra INM-5185 un valor menor a $1 \%(0,75 \%)$; baja relación del álcalis $v s \mathrm{SiO}_{2}$ $(0,03$ a 0,10 wt $\%)$ y valores de $\mathrm{K}_{2} \mathrm{O}$ bajos de $0,30 \%$ a $1,25 \%$. Las pérdidas por ignición en la mayoría de las muestras son del orden de $0,92 \%$ a $3,25 \%$, debido a la alteración moderada y deformación de las rocas a excepción de la muestra INM-5324, la cual presenta perdidas por ignición del orden de 8,06\% debido a presencia de venillas de calcita que sugieren perdidas por calcinación de $\mathrm{CO}_{2}$ (TABLA 2). 
TABLA 2. Resultados analíticos para óxidos de elementos mayores (\%wt) de las rocas analizadas de la unidad Basaltos de San Pablo.

\begin{tabular}{l|llllllllllll}
$\begin{array}{l}\text { No } \\
\text { Muestra }\end{array}$ & CM-1 & INM- & INM- & INM- & IGM- & ING- & FS-01 & INM- & FS-02 & INM- & INM- & CM-2 \\
\hline $\mathbf{S i O}_{2}$ & 51,04 & 50,78 & 53,84 & 50,31 & 49,90 & 49,61 & 43,13 & 55,36 & 44,65 & 47,95 & 45,96 & 48,84 \\
$\mathbf{A l}_{2} \mathbf{O}_{3}$ & 14,01 & 14,07 & 14,52 & 14,25 & 15,78 & 15,77 & 17,56 & 15,63 & 17,61 & 16,02 & 15,18 & 14,80 \\
$\mathbf{F e}_{2} \mathbf{O}_{3} \mathbf{T}$ & 11,29 & 11,42 & 8,97 & 10,81 & 9,65 & 9,73 & 11,23 & 6,58 & 11,35 & 10,22 & 10,24 & 10,18 \\
$\mathbf{M g O}$ & 5,85 & 6,21 & 5,43 & 7,06 & 7,76 & 7,86 & 8,00 & 8,09 & 8,11 & 6,92 & 6,82 & 9,46 \\
$\mathbf{C a O}$ & 8,10 & 7,74 & 7,65 & 8,71 & 9,88 & 10,10 & 10,50 & 6,40 & 11,45 & 13,17 & 8,51 & 7,69 \\
$\mathbf{N a}_{2} \mathbf{O}$ & 4,35 & 4,20 & 5,49 & 3,72 & 2,72 & 2,70 & 4,00 & 3,28 & 1,63 & 2,36 & 3,58 & 3,31 \\
$\mathbf{K}_{2} \mathbf{O}$ & 0,34 & 0,30 & 0,1 & 0,58 & 0,78 & 0,64 & 0,36 & 0,48 & 0,45 & 0,19 & 1,05 & 0,61 \\
$\mathbf{T i O}_{2}$ & 1,81 & 2,23 & 1,26 & 1,69 & 1,30 & 1,29 & 3,00 & 0,75 & 3,02 & 1,15 & 1,22 & 1,43 \\
$\mathbf{P}_{2} \mathbf{O}_{5}$ & 0,182 & 0,26 & 0,18 & 0,19 & 0,13 & 0,12 & - & 0,20 & - & 0,13 & 0,14 & 0,127 \\
MnO & 0,022 & 0,023 & 0,13 & 0,022 & 0,020 & 0,020 & 0,20 & 0,015 & 0,19 & 0,18 & 0,17 & 0,020 \\
LOI & 2,71 & 2,47 & 1,65 & 2,40 & 1,83 & 1,89 & 0,92 & 2,97 & 1,01 & 1,36 & 8,06 & 3,25 \\
SUMA & 99,70 & 99,71 & 99,23 & 99,74 & 99,75 & 99,73 & 98,90 & 99,75 & 99,47 & 99,65 & 100,9 & 99,72
\end{tabular}

Las rocas analizadas de la unidad Basaltos de San Pablo se distribuyen en el diagrama TAS (Le Bas et al., 1986) en los campos de basaltos y andesitas basálticas; la muestra INM-5185 alcanza el campo de las andesitas y la muestra FS-01 el campo de las basanitas de afinidad alcalina, esta última con contenido de $\mathrm{SiO}_{2}$ de $43,13 \%$. Se puede observar que la mayoría de las muestras pertenecen a la serie subalcalina (FIGURA 4). En el mismo diagrama se graficaron muestras de rocas basálticas de las unidades Diabasas de San José de Urama (asteriscos) y del Complejo Quebradagrande (equis) (FIGURA 4). Se hace evidente que las muestras de la unidad Basaltos de San Pablo poseen contenidos más elevados de álcalis y de $\mathrm{K}_{2} \mathrm{O}$.

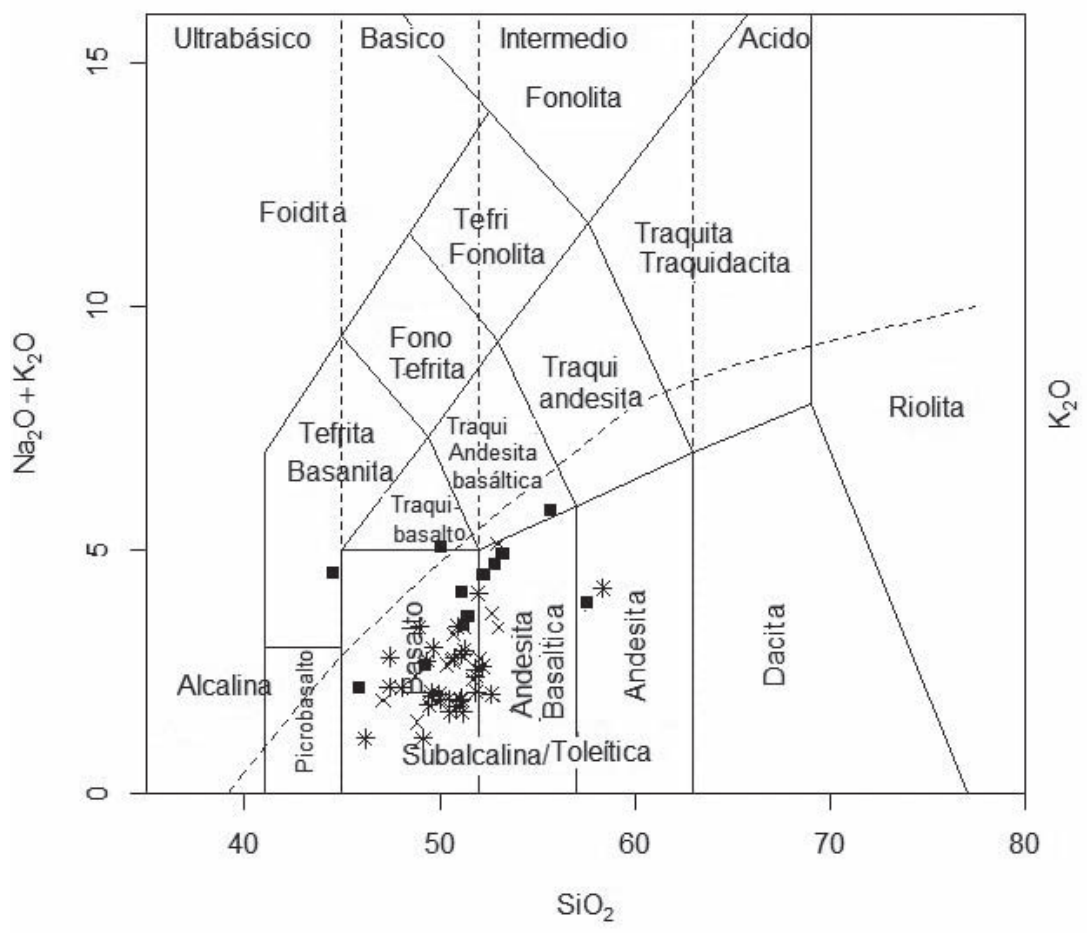

FIGURA 4. Diagrama TAS (Le Bas et al., 1986). Los cuadros representan rocas de la unidad Basaltos de San Pablo, los asteriscos (*) rocas de la unidad Diabasas de San José de Urama; y las equis (x) basaltos (diabasas) del Complejo Quebradagrande. 
El contenido de $\mathrm{K}_{2} \mathrm{O}$ de rocas de la unidad Basaltos de San Pablo es más alto que los valores de $\mathrm{K}_{2} \mathrm{O}$ de basaltos (diabasas) del Complejo Quebradagrande y que las rocas de la unidad Diabasas de San José de Urama las cuales caen principalmente dentro de la serie toleítica (Rodríguez y Arango, 2013; Rodríguez y Cetina, 2016).

En el diagrama triangular $\mathrm{Fe}^{\mathrm{t}}+\mathrm{Ti}, \mathrm{Al}, \mathrm{Mg}$ de Jensen (1976) (FIGURA 5A)-, se observa que la mayoría de rocas de la unidad Basaltos de San Pablo, el Complejo Quebradagrande y las Diabasas de San José de Urama se concentran en el campo de la serie de basaltos toleíticos ricos en $\mathrm{Mg}$, con algunas pocas muestras dentro del campo de los basaltos toleíticos ricos en Fe.

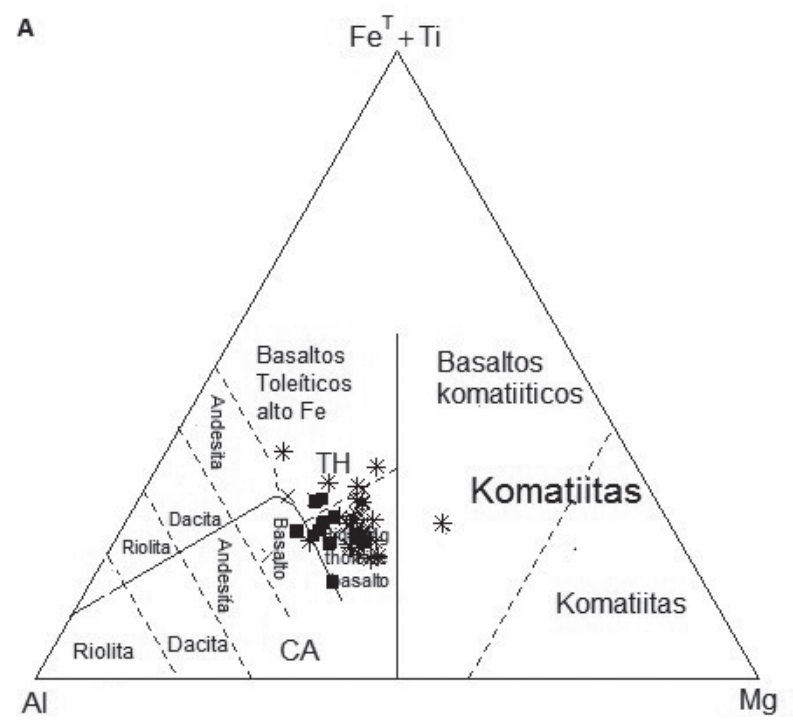

Al utilizar el diagrama AFM de Irvine y Baragar (1971) (FIGURA 5B) se puede observar que las rocas de la unidad Basaltos de San Pablo pertenecen a la serie calcoalcalina poco diferenciada, con enriquecimiento de hierro en el estadio temprano de diferenciación y únicamente las muestras FS-02 y INM-6180 caen en el campo toleítico, en general con una diferenciación paralela al de basaltos de series toleíticas, pero dentro del campo calcoalcalino; por el contrario los basaltos (diabasas) del Complejo Quebradagrande y las Diabasas de San José de Urama se distribuyen en su mayoría en el campo de las rocas toleíticas poco diferenciadas con bajo contenido de álcalis.

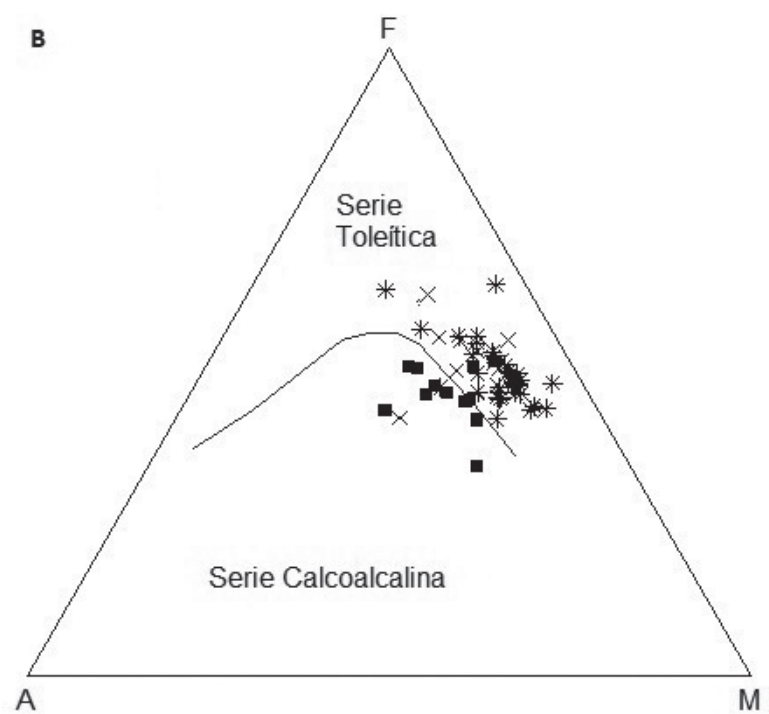

FIGURA 5. A. Diagrama triangular Fet+Ti, Al, Mg (Jensen, 1976). B. Diagrama AFM según Irvine y Baragar (1971) para muestras de basaltos de las unidades Basaltos de San Pablo (cuadros), Complejo Quebradagrande (cruces - x) y Diabasas de San José de Urama (asteriscos - *).

En la TABLA 3 se presentan los resultados de las concentraciones de elementos traza y tierras raras (REE) de las muestras analizadas de la unidad Basaltos de San Pablo. Las concentraciones de elementos compatibles como Ni están entre 70 y 179 ppm, los valores de V entre 228 y 295 ppm, con disminución de la concentración de $\mathrm{V}$ con el aumento de $\mathrm{SiO}_{2}$ y la muestra INM-5185 tiene un valor menor de $\mathrm{V}$ de 125,3 ppm. Los valores de $\mathrm{Cr}$ en los Basaltos de San Pablo varían entre 170 y 436 ppm, excepto la muestra FS-01 que tiene un valor de $\mathrm{Cr}<100 \mathrm{ppm}$.

Las rocas de la unidad Basaltos de San Pablo presentan valores de Sr entre 96,8 y 212 ppm y la muestra INM5185 tiene un valor mayor de Sr de 298,6 ppm; los valores de $\mathrm{Nb}$ varían entre 0,5 y 6,2 ppm, el contenido de
Zr está en el rango de 34,6 a 136 ppm, la mayoría de las muestras presentan valores de $\mathrm{K}_{2} \mathrm{O}$ entre 0,3 y 1,25\%, valores más altos que en las rocas basálticas de corteza oceánica de San José de Urama y Quebradagrande (de acuerdo a los datos de Rodríguez y Arango, 2013 y Rodríguez y Cetina, 2016); los contenidos de Ni están en el rango entre 70 y 457 ppm relativamente altos, siendo en general la mayoría de los valores similares a los encontrados en cortezas oceánicas de tipo MORB y arcos de isla derivados de rocas del manto. Al comparar los valores de $\mathrm{K}_{2} \mathrm{O}$, Sr, $\mathrm{Nb}, \mathrm{Zr}$ de los basaltos de la unidad Basaltos de San Pablo con los contenidos y valores típicos de rocas generadas en dorsales mesooceánicas presentados por Wilson (1989), se observa que los Basaltos de San Pablo poseen valores más elevados en $\mathrm{K}_{2} \mathrm{O}$ con relación a los valores para basaltos 
tipo MORB, pero similares a los de las rocas generadas en arcos de isla (Winter, 2001), los contenidos de Sr son variables, el contenido de $\mathrm{Zr}$ está dentro del rango de los basaltos tipo MORB, es similar a los basaltos (diabasas) del Complejo Quebradagrande y presentan valores más altos que los basaltos de Plateau de las
Diabasas de San José de Urama. Las relaciones $\mathrm{Zr} / \mathrm{Y}$, $\mathrm{Nb} / \mathrm{Y}$ y $(\mathrm{La} / \mathrm{Yb})_{\mathrm{N}}$ están dentro del rango de los basaltos tipo MORB presentados por Wilson (1989); los valores de la relación Ti/V son variables, pero alrededor de 30, más altos que en las rocas generadas en arcos de islas.

TABLA 3. Contenido de elementos traza y tierras raras en rocas analizadas de la unidad Basaltos de San Pablo.

\begin{tabular}{|c|c|c|c|c|c|c|c|c|c|c|c|c|}
\hline $\begin{array}{c}\text { No. } \\
\text { Muestra }\end{array}$ & CM-1 & CM-2 & FS-01 & FS-02 & $\begin{array}{l}\text { INM- } \\
5185\end{array}$ & $\begin{array}{l}\text { "INM- } \\
5271\end{array}$ & $\begin{array}{l}\text { INM- } \\
5312\end{array}$ & $\begin{array}{l}\text { "INM- } \\
5324\end{array}$ & $\begin{array}{l}\text { INM- } \\
5365\end{array}$ & $\begin{array}{l}\text { *INM- } \\
6180\end{array}$ & $\begin{array}{l}\text { IGM- } \\
6816\end{array}$ & $\begin{array}{l}\text { IGM- } \\
6772\end{array}$ \\
\hline Sc & 71,3 & 76,0 & 43,7 & 44,7 & 24,9 & 39,7 & 46,2 & 42,0 & 46,5 & 34,0 & 41,9 & 39,4 \\
\hline Co & 44,4 & 57,9 & 44,6 & 51,9 & 42,5 & 40,0 & 56,1 & 51,0 & 58,7 & 44,8 & 60,0 & 60,7 \\
\hline $\mathrm{Ni}$ & 79,1 & 102,6 & 69,8 & 108,7 & 179,5 & 281,7 & 70,5 & 41,7 & 82,2 & 460,1 & 129,5 & 114,0 \\
\hline $\mathrm{Cu}$ & 46,9 & 86,2 & 69,2 & 81,2 & 20,5 & 56,8 & 66,2 & 64,1 & 60,1 & 53,4 & 86,3 & 73,3 \\
\hline $\mathrm{Zn}$ & 131,8 & 113,3 & 78,2 & 88,6 & 102,9 & 125,1 & 135,2 & 108,4 & 167,7 & 128,1 & 106,3 & 103,0 \\
\hline $\mathrm{Ga}$ & 22,4 & 20,4 & 15,8 & 17,4 & 19,1 & 10,6 & 20,3 & 17,4 & 26,0 & 13,2 & 20,8 & 19,9 \\
\hline As & 2,5 & 3,2 & 3,1 & 2,4 & 1,1 & & 1,4 & & 1,6 & & 1,4 & 1,2 \\
\hline $\mathrm{Rb}$ & 12,2 & 9,3 & 4,3 & 8,0 & 16,8 & 0,9 & 15,9 & 33,1 & 13,8 & 0,9 & 30,5 & 39,0 \\
\hline $\mathrm{Sr}$ & 104,4 & 191,8 & 122,8 & 151,2 & 298,6 & 96,8 & 188,1 & 124,3 & 195,7 & 113,7 & 208,0 & 211,4 \\
\hline Y & 44,4 & 32,0 & & & 16,4 & 28,6 & 39,2 & 28,0 & 55,3 & 22,5 & 30,9 & 31,2 \\
\hline $\mathrm{Cd}$ & 0,3 & 0,4 & 0,1 & 0,2 & 0,3 & & 0,2 & & 0,4 & & 0,1 & 0,1 \\
\hline Cs & 17,9 & 3,3 & 0,1 & 0,8 & 1,7 & 0,2 & 0,5 & 2,0 & 0,9 & 0,2 & 0,9 & 1,1 \\
\hline $\mathrm{Ba}$ & 86,3 & 46,3 & 31,3 & 74,7 & 530,5 & 205,5 & 122,2 & 157,8 & 73,7 & 11,6 & 114,0 & 135,2 \\
\hline $\mathrm{La}$ & 6,5 & 4,1 & 5,4 & 4,8 & 10,5 & 1,9 & 10,0 & 2,5 & 13,3 & 1,8 & 4,4 & 4,8 \\
\hline $\mathrm{Ce}$ & 16,7 & 11,2 & 11,2 & 10,3 & 23,3 & 6,6 & 24,8 & 7,9 & 34,8 & 5,8 & 12,3 & 13,0 \\
\hline $\operatorname{Pr}$ & 3,1 & 2,1 & 1,8 & 1,7 & 3,3 & 1,2 & 3,8 & 1,4 & 5,7 & 1,1 & 2,2 & 2,3 \\
\hline $\mathrm{Nd}$ & 17,0 & 11,6 & 9,3 & 8,6 & 13,9 & 6,9 & 20,0 & 7,5 & 28,0 & 5,7 & 11,2 & 12,4 \\
\hline $\mathrm{Sm}$ & 5,4 & 3,9 & 3,2 & 3,0 & 3,3 & 2,4 & 5,7 & 2,6 & 8,4 & 2,0 & 3,8 & 3,9 \\
\hline $\mathrm{Eu}$ & 1,2 & 0,9 & 1,2 & 1,2 & 1,3 & 0,8 & 1,8 & 0,8 & 2,4 & 0,6 & 1,3 & 1,4 \\
\hline Gd & 5,7 & 4,1 & 3,5 & 3,2 & 3,1 & 3,1 & 5,7 & 3,1 & 8,5 & 2,3 & 4,1 & 4,2 \\
\hline $\mathrm{Tb}$ & 1,3 & 0,9 & 0,9 & 0,8 & 0,5 & 0,6 & 1,2 & 0,6 & 1,7 & 0,4 & 0,9 & 0,9 \\
\hline Dy & 8,1 & 5,8 & 5,3 & 4,8 & 3,1 & 4,5 & 7,4 & 4,5 & 10,7 & 3,4 & 5,7 & 5,8 \\
\hline Ho & 1,7 & 1,2 & 1,1 & 1,0 & 0,7 & 1,0 & 1,6 & 0,9 & 2,3 & 0,7 & 1,3 & 1,3 \\
\hline Er & 4,9 & 3,5 & 3,4 & 3,0 & 1,9 & 2,6 & 4,6 & 2,6 & 6,1 & 2,0 & 3,6 & 3,5 \\
\hline $\mathrm{Tm}$ & 0,7 & 0,5 & 0,5 & 0,4 & 0,3 & 0,4 & 0,6 & 0,4 & 0,9 & 0,3 & 0,5 & 0,5 \\
\hline $\mathrm{Yb}$ & 4,3 & 3,1 & 3,1 & 2,7 & 1,7 & 2,7 & 4,1 & 2,7 & 5,2 & 2,1 & 3,1 & 3,1 \\
\hline $\mathrm{Lu}$ & 0,6 & 0,5 & 0,5 & 0,4 & 0,3 & 0,4 & 0,6 & 0,4 & 0,7 & 0,3 & 0,5 & 0,5 \\
\hline $\mathrm{Pb}$ & 5,3 & 5,2 & 9,7 & 5,0 & 3,3 & 1,5 & $<2,0$ & 2,8 & 2,7 & 1,3 & 2,2 & $<2,0$ \\
\hline Th & 0,7 & 0,1 & 1,2 & 1,1 & 1,9 & 0,6 & 1,1 & 0,4 & 1,5 & 0,2 & 0,4 & 0,4 \\
\hline $\mathrm{U}$ & 0,4 & 0,1 & 0,2 & 0,2 & 0,6 & 0,1 & 0,2 & 0,1 & 0,8 & 0,0 & 0,1 & 0,1 \\
\hline V & 295,3 & 263,0 & & & 125,3 & 253,2 & 249,4 & 271,8 & 273,5 & 239,1 & 236,9 & 234,2 \\
\hline $\mathrm{Cr}$ & 170,2 & 260,6 & 297,5 & 435,8 & 326,8 & 378,3 & 95,0 & 282,5 & 130,4 & 397,3 & 254,4 & 234,0 \\
\hline $\mathrm{Zr}$ & 122,6 & 85,4 & & & 97,4 & 58,1 & 136,5 & 72,3 & 190,5 & 34,6 & 78,1 & 82,2 \\
\hline $\mathrm{Nb}$ & 3,3 & 2,4 & 2,2 & 2,0 & 4,5 & 1,3 & 6,2 & 1,3 & 6,2 & 0,5 & 2,4 & 2,7 \\
\hline Тa & 0,0 & & 0,0 & 0,0 & 16,8 & 0,2 & 12,0 & 0,2 & 0,0 & 0,1 & 12,9 & 8,1 \\
\hline $\mathrm{Hf}$ & & & & & & 1,6 & & 1,8 & & 0,8 & & \\
\hline
\end{tabular}

* Muestras analizadas en la Universidad de Cardiff 
La muestra INM-5185 presenta relaciones de $(\mathrm{La} / \mathrm{Yb})_{\mathrm{N}} \mathrm{y}$ $(\mathrm{La} / \mathrm{Sm})_{\mathrm{N}}$ más altas que las demás muestras, del orden de 4,2 y 1,96 respectivamente y un mayor \#Mg (de
70,9), mientras en el resto de muestras el \#Mg varía entre 50 y 64 (TABLA 4).

TABLA 4. Relaciones de elementos traza en muestras de basaltos de la unidad Basaltos de San Pablo.

\begin{tabular}{|c|c|c|c|c|c|c|c|c|}
\hline Muestra & $\mathbf{E u} / \mathbf{E u} *$ & $(\mathrm{La} / \mathrm{Yb}) \mathrm{N}$ & $(\mathbf{L a} / \mathbf{S m}) \mathbf{N}$ & $(\mathrm{Ce} / \mathrm{Yb}) \mathbf{N}$ & $(\mathrm{Ce} / \mathrm{Sm}) \mathrm{N}$ & $(\mathbf{E u} / \mathbf{Y b}) \mathbf{N}$ & Sum_REE & \#Mg \\
\hline CM-2 & 0,7 & 0,9 & 0,6 & 0,92 & 0,67 & 0,87 & 53,53 & 64,8 \\
\hline INM-5185 & 1,2 & 4,2 & 1,9 & 3,53 & 1,64 & 2,19 & 67,03 & 70,9 \\
\hline ING-6816 & 1,0 & 1,0 & 0,7 & 1,01 & 0,75 & 1,23 & 54,94 & 61,5 \\
\hline IGM-6772 & 1,0 & 1,1 & 0,8 & 1,07 & 0,77 & 1,28 & 57,55 & 61,4 \\
\hline INM-5312 & 1,0 & 1,6 & 1,1 & 1,56 & 1,02 & 1,27 & 92,1 & 56,4 \\
\hline INM-5365 & 0,9 & 1,7 & 1,0 & 1,7 & 0,97 & 1,3 & 128,64 & 51,9 \\
\hline CM-1 & 0,7 & 1,0 & 0,7 & 0,99 & 0,73 & 0,78 & 77,15 & 50,7 \\
\hline FS-01 & 1,1 & 1,1 & 1,0 & 0,91 & 0,81 & 1,12 & 50,37 & 58,5 \\
\hline FS-02 & 1,2 & 1,2 & 1,0 & 0,97 & 0,82 & 1,32 & 45,73 & 58,6 \\
\hline INM-5271 & 0,9 & 0,5 & 0,5 & 0,63 & 0,65 & 0,84 & 34,95 & 54,5 \\
\hline INM-5324 & 0,9 & 0,6 & 0,6 & 0,75 & 0,73 & 0,89 & 37,94 & 56,9 \\
\hline INM-6180 & 0,9 & 0,6 & 0,5 & 0,7 & 0,67 & 0,83 & 28,69 & 57,3 \\
\hline
\end{tabular}

La relación $(\mathrm{La} / \mathrm{Sm})_{\mathrm{N}}$ para las rocas de la unidad Basaltos de San Pablo, es menor o igual a 1 y únicamente la muestra INM-5185 tiene una relación $(\mathrm{La} / \mathrm{Sm})_{\mathrm{N}}$ cercana a 2; para basaltos (diabasas) del Complejo Quebradagrande esta relación también es menor a 1 , sugiriendo que ambos grupos de basaltos son afines con basaltos tipo N-MORB (basaltos de dorsales oceánicas normales o empobrecidos), mientras que las Diabasas de San José de Urama presentan valores de $(\mathrm{La} / \mathrm{Sm})_{\mathrm{N}}$ menores y mayores a 1 (Rodríguez y Cetina, 2016; Rodríguez y Arango, 2013), comparables con ambientes de pluma, E-MORB o T-MORB. La relación Ba/La es más elevada que en los basaltos tipo MORB y OIB y comparable con los basaltos de arcos de isla toleíticos, lo que según Wilson (1989) es debido, en las zonas de subducción, al enriquecimiento en Ba proveniente de los sedimentos involucrados en el proceso de subducción. La relación $(\mathrm{La} / \mathrm{Yb})_{\mathrm{N}}$ varía entre 0,5 y 1,6 (según la TABLA 4) comparable con rocas de corteza oceánica MORB y únicamente la muestra INM-5185 presenta un valor de 4,17 más cercana a rocas de arco primitivo.

En el diagrama de REE normalizados con respecto al condrito (valores de normalización de Nakamura, 1974), las muestras de la unidad Basaltos de San Pablo presentan tres diferentes patrones (FIGURA 6): 1- las muestras INM-5324, INM-5771, INM-6180, INM5271, IGM-6816, IGM-6772, CM-2, CM-1, FS-01, FS-02 tienen un comportamiento paralelo (muestras en color negro), con un patrón con empobrecimiento en tierras raras livianas (La, Ce) (LREE) similar al patrón de basaltos tipo N-MORB (FIGURA 6A), el cual presenta enriquecimiento ligeramente mayor a 10 veces el condrito, y una tendencia plana horizontal hacia las HREE, con anomalía negativa de Eu para las rocas con mayor contenido de $\mathrm{SiO}_{2}$, anomalía que puede ser asociada a la remoción de plagioclasa. Las muestras INM-5312 y INM-5365 (en color verde) corresponden a las rocas geoquímicamente más diferenciadas, presentan una pendiente suave negativa desde las tierras raras livianas (LREE) hacia las tierras raras pesadas (HREE), conservan el paralelismo en las HREE con relación a las muestras menos diferenciadas o primitivas y tienen un comportamiento similar al de las rocas de arcos de islas bajos en K. La muestra INM-5185 presenta pendiente negativa más pronunciada que atraviesa el patrón de las demás muestras, con empobrecimiento desde las LREE hacia las HREE similar a la tendencia que presentan las rocas generadas en ambiente de arco.

En el diagrama multielemental de elementos trazas, las muestras de la unidad Basaltos de San Pablo presentan mayor dispersión en los contenidos de los elementos más móviles (LILE), con anomalías positivas en Cs, Ba, $\mathrm{K}$ y $\mathrm{Pb}$, un suave empobrecimiento para los elementos traza más inmóviles (HFSE), con patrones ligeramente descendentes entre La y Lu, y anomalías negativas de $\mathrm{Nb}$ y Zr que sugieren ambiente de arco (FIGURA 6B 
y FIGURA 6C). La muestra INM-5185 tiene un mayor empobrecimiento de los elementos traza inmóviles (Ti, Y, Yb, Dy, Lu) con relación a las demás muestras analizadas, presenta anomalía negativa de $\mathrm{Nb}$ con respecto a Th - Cs y pendiente negativa más alta que las demás muestras, atravesando los patrones de las otras rocas, sugiriendo que es una roca generada en un ambiente de arco, probablemente en un evento diferente al de las otras rocas.
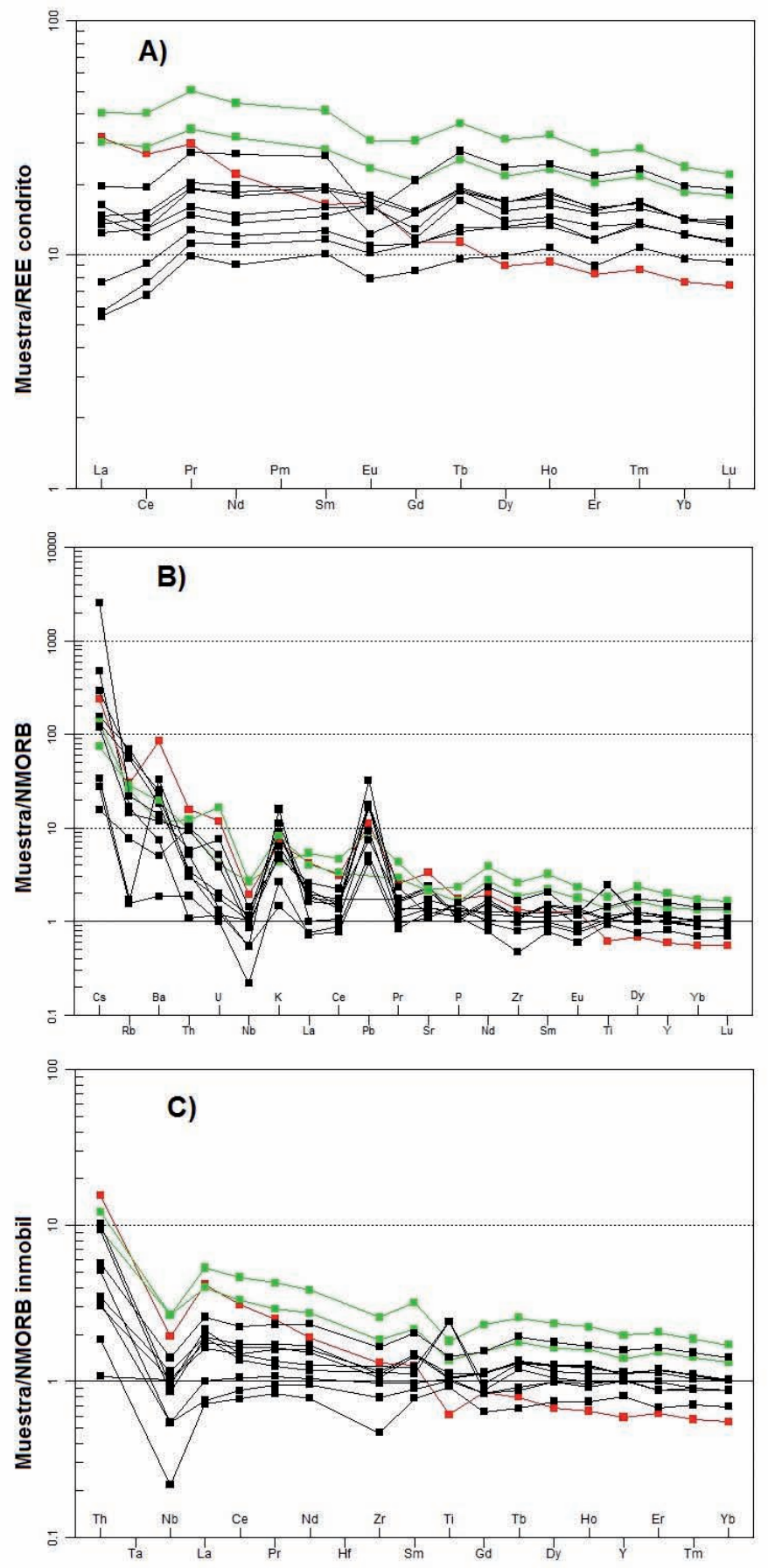

FIGURA 6. Diagramas multielementales para muestras de los Basaltos de San Pablo. A. Diagramas de tierras raras (REE) normalizados con respecto al condrito (McDonough y Sun, 1995). B. Diagramas multielementales normalizados respecto al N-MORB (Sun y McDonough, 1989). C. Diagramas multielementales normalizados respecto al N-MORB para elementos traza inmobiles (Sun y McDonough, 1989 en Pearce, 2014). 
En la FIGURA 7 y la TABLA 5 se comparan los patrones de REE para una muestra de basaltos de la unidad Basaltos de San Pablo, con una muestra de las Diabasas de San José de Urama y una diabasa del Complejo Quebradagrande(INM-5324; IGM-700114, IGM-7585). Las tres muestras tienen contenidos similares de $\mathrm{MgO}$ (7,41\%, 7,67\% y 7,79\% respectivamente). La muestra INM-5324 de los Basaltos de San Pablo, aunque tiene un alto valor de LOI (perdidas por calcinación), debido a la presencia de venas de calcita, no muestra alteración de la plagioclasa y el piroxeno y el patrón es paralelo al de otras muestras con contenidos de MgO menores y mayores de la unidad Basaltos de San Pablo; tiene un patrón similar al de la muestra IGM-7585 del Complejo Quebradagrande que está más enriquecida en las REE, con empobrecimiento en las tierras raras livianas, y el patrón es similar al de los basaltos de tipo N-MORB en las rocas más primitivas. La muestra de basalto de la unidad de Diabasas de San José de Urama presenta un patrón plano similar a los T-MORB (plateau). La relación $(\mathrm{La} / \mathrm{Yb})_{\mathrm{N}}$ en la muestra INM-5324 es de 0,62, en el basalto (diabasa) del Complejo Quebradagrande es de 0,75 (IGM-7585) y en la diabasa de San José de Urama es de 1,02, valores que son similares a los de diferentes cortezas oceánicas. Las muestras INM5324 e IGM-7585 indican fuentes diferentes, con un patrón subparalelo pero más empobrecido en la muestra de basalto de la unidad Basaltos de San Pablo, siendo la muestra de basalto (diabasa) del Complejo Quebradagrande proveniente de una fuente menos primitiva. La muestra IGM-706399 de las Diabasas de San José de Urama tiene un patrón no paralelo a las dos muestras anteriores.

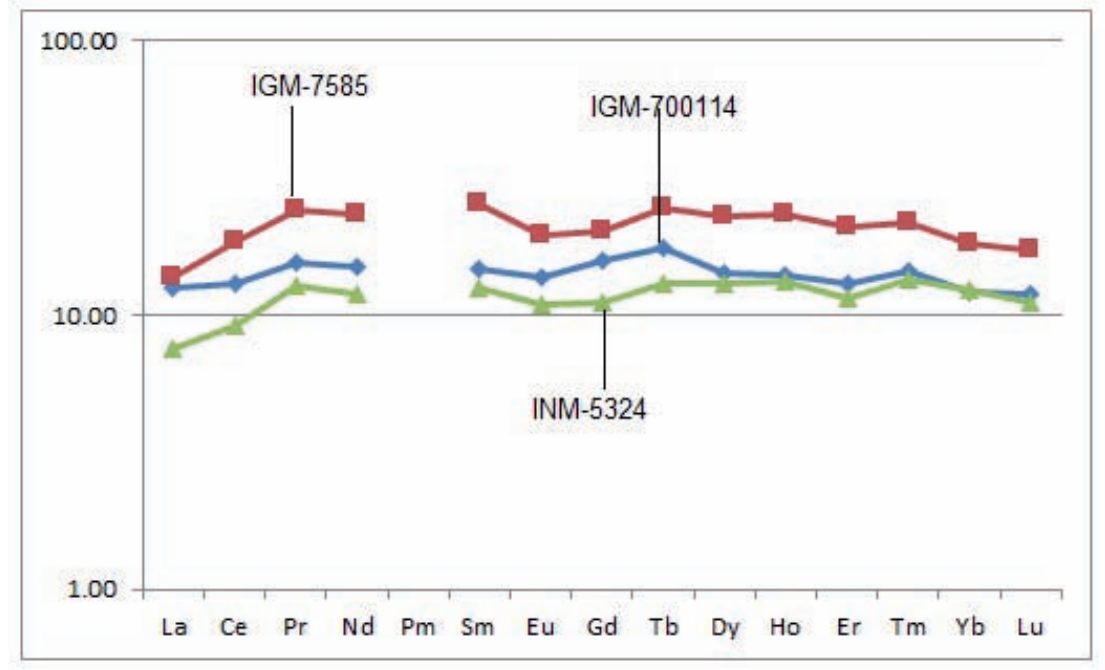

FIGURA 7. Patrón de tierras raras para muestras de basaltos de la unidad Basaltos de San Pablo (verde), Complejo Quebradagrande (Rojo) y Diabasas de San José de Urama (Azul). En rojo muestra-IGM-7585- Complejo Quebradagrande, en verde - INM-5324 Basaltos de San Pablo y en azul muestra IGM-700114- Diabasas de San José de Urama.

TABLA 5. Valores de tierras raras normalizados de tres muestras de diabasa con valores de MgO comparables. Muestra INM5324 Basaltos de San Pablo, muestra 706399 Diabasas de San José de Urama y Muestra 80729 basalto (diabasa) del Complejo Quebradagrande.

\begin{tabular}{|c|c|c|c|c|c|c|c|c|c|c|c|c|c|c|c|c|}
\hline MUESTRA & $\mathbf{L a}$ & Ce & Pr & Nd & Sm & Eu & Gd & Tb & Dy & Ho & Er & $\mathrm{Tm}$ & $\mathbf{Y b}$ & Lu & $\begin{array}{l}\mathbf{L a} / \\
\mathbf{Y b}\end{array}$ & $\% \mathrm{MgO}$ \\
\hline $\begin{array}{c}\text { Valor de } \\
\text { normalización }\end{array}$ & 0,33 & 0,87 & 0,11 & 0,63 & 0,2 & 0,08 & 0,28 & 0,05 & 0,34 & 0,07 & 0,23 & 0,03 & 0,22 & 0,03 & & \\
\hline 700114 & 12,46 & 12,95 & 15,54 & 15,08 & 14,63 & 13,77 & 15,83 & 17,45 & 14,23 & 14 & 13,11 & 14,43 & 12,18 & 11,98 & 1,02 & 7,67 \\
\hline 7585 & 13,6 & 18,57 & 24,18 & 23,23 & 25,4 & 19,56 & 20,37 & 24,69 & 22,71 & 23,36 & 21,01 & 21,86 & 18,22 & 17,29 & 0,75 & 7,79 \\
\hline 5324 & 7,6 & 9,14 & 12,69 & 11,98 & 12,59 & 10,92 & 11,07 & 13,03 & 13,1 & 13,25 & 11,57 & 13,36 & 12,24 & 11,2 & 0,62 & 7,41 \\
\hline
\end{tabular}


La pareja geoquímica $\mathrm{Th}-\mathrm{Nb}$ es particularmente importante para la identificación y clasificación de los basaltos oceánicos y por lo tanto para la demostración de un ambiente oceánico diferente a subducción (Pearce, 2008). El gráfico $\mathrm{Th} / \mathrm{Yb}-\mathrm{Nb} / \mathrm{Yb}$ permite definir el carácter oceánico de diferentes tipos de basaltos, los cuales grafican dentro del campo diagonal MORB-OIB de la FIGURA 8, con el eje principal de dispersión a lo largo de este campo. Los basaltos formados en márgenes continentales y en las zonas de subducción son comúnmente localizados dentro del gráfico por encima del campo MORB-OIB y prácticamente todos los magmas de arco grafican por encima del campo MORB-OIB (Pearce y Peate, 1995), aunque algunas lavas de cuencas de retro arco pueden aparecer por encima de este campo (Pearce, 2008).

En la FIGURA 8 se grafican las rocas de la unidad Basaltos de San Pablo, los basaltos (diabasas) del Complejo Quebradagrande (Rodríguez y Cetina, 2016) y las Diabasas de San José de Urama (tomados de Rodríguez y Arango, 2013).

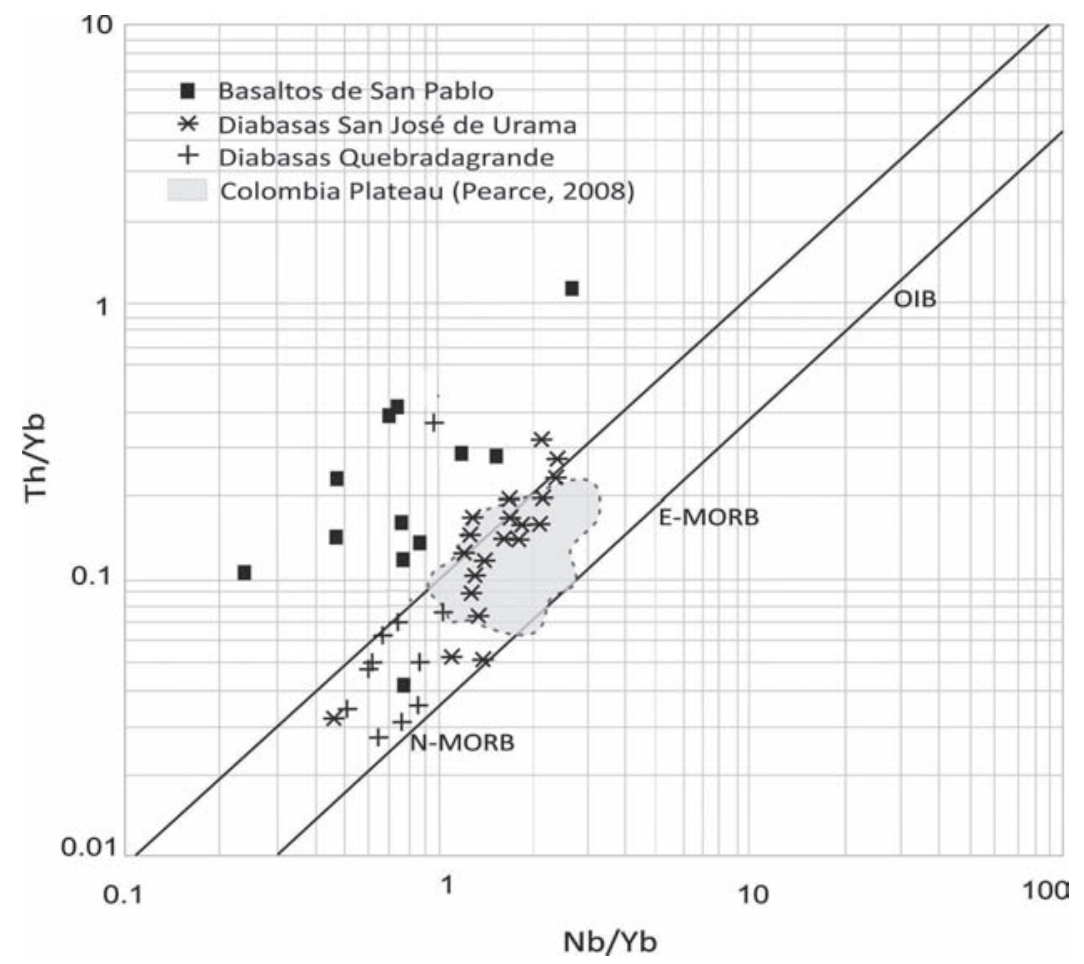

FIGURA 8. Discriminación de ambiente tectónico para muestras de la unidad Basaltos de San Pablo a partir del diagrama de Pearce (1982). Manto empobrecido MORB- N. manto enriquecido. MORB-E. Basaltos de islas oceánicas (OIB). La línea punteada representa el Plateau Colombia- según datos de Pearce (2008).

Las rocas de la unidad Basaltos de San Pablo grafican por encima del campo (MORB-OIB), resultado que corrobora que estos basaltos se formaron en arcos de isla o cuencas de back-arc. Una sola muestra de basalto de esta unidad (CM-1) gráfica dentro del campo de las rocas de corteza oceánica, hacia los basaltos oceánicos de tipo N-MORB y se ubica junto a las muestras de diabasas del Complejo Quebradagrande. Las muestras de la unidad Diabasas de San José de Urama grafican entre los basaltos N-MORB y E-MORB, en el campo de los basaltos de corteza oceánica y dentro del campo del plateau Colombia delimitado por Pearce (2008), siendo las rocas de esta unidad generadas en este plateau. Las muestras de la unidad Basaltos de San Pablo presentan relaciones $\mathrm{Nb} / \mathrm{Yb}$ similares y relaciones $\mathrm{Th} / \mathrm{Yb}$ mayores a los basaltos (diabasas) del Complejo Quebradagrande y corresponden a basaltos de arco formados en un ambiente de subducción oceánica, donde a las rocas de arco se le adiciona Th móvil proveniente de la subducción y se desplazan los valores por encima del campo MORB-OIB (Pearce et al., 2005). 


\section{DISCUSIÓN}

\section{Localización tectónica de los Basaltos de San Pablo}

La unidad Basaltos de San Pablo, conforman un bloque tectónico localizado en la parte más norte del núcleo de la Cordillera Central, al oriente de las fallas de San Jerónimo y Espíritu Santo y al occidente de las fallas de Palestina-Otú-Pericos y Liberia, siendo una unidad volcánica con una secuencia sedimentaria marina asociada, particular en su posición geotectónica dentro de la Cordillera Central de Colombia (FIGURA 1).

La unidad Basaltos de San Pablo no se correlaciona con los basaltos de corteza oceánica del Complejo Quebradagrande y las rocas de plateau de la Cordillera Occidental (Diabasas de San José de Urama - Rodríguez y Arango, 2013), si se analiza la posición geotectónica de las tres unidades: el Complejo Quebradagrande, en el sentido de Maya y González (1995), corresponde a un litodema localizado entre las fallas San Jerónimo al oriente y Silvia-Pijao al occidente, el cual está en contacto fallado con los complejos Arquía (al oeste) y Cajamarca (al este), en una posición geotectónica más occidental que la unidad de Basaltos de San Pablo. Las Diabasas de San José de Urama afloran en el norte de la Cordillera Occidental, entre las fallas Cauca - Almaguer al oriente y las fallas de Uramita y Dabeiba-Pueblo Rico al occidente; esta unidad se encuentra en contacto fallado con el Bloque ChocóPanamá (al oeste) y con el Complejo Arquía (al este), en una posición geotectónica más occidental que el Complejo Quebradagrande y separado de éste por el Complejo Arquía y separado de la unidad Basaltos de San Pablo por los complejos Arquía, Quebradagrande y Cajamarca.

Las rocas alrededor del bloque que conforma la unidad Basaltos de San Pablo, corresponden a esquistos cuarzo sericíticos, cuarcitas, esquistos verdes, neises y anfibolitas, agrupadas en el Complejo Cajamarca (González, 2001). En este sector de la Cordillera Central se han reconocido en los últimos diez años rocas con metamorfismo regional dinamo térmico con edades de metamorfismo del Paleozoico, Triásico, Jurásico superior y Cretácico, de acuerdo con los datos publicados por Vinasco et al. (2006); Restrepo et al. (2009, 2011, 2012), Blanco-Quintero et al. (2014), Rodríguez y Correa-Martínez (2015) y Rodríguez et al. (2016), sin que a la fecha se conozca con certeza la distribución espacial de las unidades metamórficas de diferente edad y con cuales de ellas se encuentra en contacto fallado la unidad Basaltos de San Pablo, que regionalmente se posiciona entre las rocas metamórficas del Complejo Cajamarca en el sentido de Maya y González (1995).

La unidad Basaltos de San Pablo se encuentra intruída por rocas del Batolito Antioqueño (Hall et al., 1972), que corresponde a un plutón tonalítico generado en un arco de margen continental (Leal-Mejía, 2011), con edades entre 70 y $89 \mathrm{Ma}$, que post-data la unidad Basaltos de San Pablo, sin que hasta el momento se tengan dataciones de los basaltos, ni descripción de fósiles que definan la edad de los sedimentos marinos intercalados. En este trabajo se trató de datar dos muestras por el método Ar-Ar de basaltos de la unidad Basaltos de San Pablo, en los laboratorios de la Universidad de Oregón (EEUU), muestras que presentaron un resultado negativo.

La posición geotectónica de las rocas de la unidad Basaltos de San Pablo no tiene comparación con otras unidades volcánicas de la Cordillera Central, es compleja de explicar entre los terrenos metamórficos de la Cordillera Central, que han sido llamados, Anacona (Paleozoico), Tahamí (Triásico), Tierradentro (Jurásico Superior) (Restrepo et al., 2009; Martens et al., 2014; Rodríguez et al., 2017). La unidad Basaltos de San Pablo corresponde a un bloque fallado con las rocas metamórficas, pero no se sabe estas rocas a cual o cuales de estos terrenos corresponden.

\section{Composición química y ambiente geotectónico}

Los basaltos de la unidad Basaltos de San Pablo tienen afinidad subalcalina - calcoalcalina, afinidad que corresponde a rocas generadas por magmas que están restringidos a zonas de subducción (Winter, 2001). La similitud en el patrón entre los basaltos más primitivos de la unidad Basaltos de San Pablo, con el patrón de basaltos oceánicos empobrecidos de tipo N-MORB, en el diagrama multielemental de tierras raras vs el condrito de Nakamura (1974), sugiere que el arco probablemente se desarrolló a partir de la interacción entre cortezas oceánicas de tipo N-MORB y que a medida que evolucionó, generó rocas menos primitivas que tienen un patrón con ligera pendiente negativa de las REE, similar al de rocas generadas en arcos de isla con contenido bajo de K (FIGURA 6A).

Las rocas de la Unidad Basaltos de San Pablo tienen anomalía negativa de Nb con relación a Th y anomalías positivas de $\mathrm{K}$ y $\mathrm{Pb}$ (diagrama multielemental de elementos traza - FIGURAS 6B y 6C), además, presenta enriquecimiento en Th y relaciones más altas 
de $\mathrm{Th} / \mathrm{Yb}$ que las rocas de corteza oceánica (MORB), ubicando las rocas dentro del campo de los arcos de islas (FIGURA 8), además, presentan enriquecimiento progresivo en los elementos traza más incompatibles (es decir, LREE y Th) y un contenido de Nb más bajo que el de las rocas de corteza oceánica.

Las rocas de la unidad Basaltos de San Pablo tienen valores más altos de $\mathrm{K}_{2} \mathrm{O}$ y álcalis (concordante con su afinidad a la serie calcoalcalina), que los basaltos de corteza oceánica (diabasas) del Complejo Quebradagrande y que los basaltos (diabasas) de la unidad Diabasas de San José de Urama, de acuerdo a los datos publicados por Rodríguez y Cetina (2016) y Rodríguez y Arango (2013), que grafican dentro de la serie toleítica. Los contenidos de $\mathrm{TiO}_{2}$ de la unidad Basaltos de San Pablo son similares a los basaltos de corteza oceánica (diabasas) del Complejo Quebradagrande $\left(\mathrm{TiO}_{2}\right.$ entre $1 \%$ y $\left.2 \%\right)$ y presenta valores más altos de $\mathrm{TiO}_{2}$ que las rocas de la unidad Diabasas de San José de Urama, que tiene $\mathrm{TiO}_{2}<1 \%$.

Los mayores contenidos de Th en las rocas basálticas de la unidad Basaltos de San Pablo sugieren aporte de este elemento proveniente de la subducción, debido a contaminación por sedimentos introducidos sobre la placa subducida, que hacen que las rocas se desplacen por encima del campo MORB-OIB en el diagrama de Pearce (1982), indicando que las rocas de la unidad Basaltos de San Pablo tienen influencia de subducción, con valores de $(\mathrm{La} / \mathrm{Yb})<2$ normales en rocas de corteza oceánica, sugiriendo que se trata de basaltos relacionados a un ambiente de subducción probablemente entre cortezas oceánicas de tipo N-MORB.

\section{CONCLUSIONES}

La unidad Basaltos de San Pablo está constituida por basaltos con texturas ofíticas, subofíticas, intersectales, intergranulares y variolíticas, petrográficamente comparables con los basaltos (diabasas) de corteza oceánica del Complejo Quebradagrande y las Diabasas de San José de Urama, sin que se observen diferencias macroscópicas y microscópicas importantes entre las rocas de las tres unidades.

La unidad Basaltos de San Pablo presenta características geoquímicas que sugieren que se desarrolló a partir de la interacción entre cortezas oceánicas de tipo $\mathrm{N}$-MORB, dentro de un ambiente de arco de islas poco evolucionado.
En el noroccidente de los Andes de Colombia se reconocen tres unidades de basaltos (diabasas) con características macroscópicas y microscópicas similares, formadas en ambientes geotectónicos diferentes, como sugiere la composición litogeoquímica de cada una de ellas: la unidad Basaltos de San Pablo se relacionan con un ambiente de arco de islas poco diferenciado, con sedimentos marinos asociados; los basaltos (diabasas) del Complejo Quebradagrande químicamente se asemejan a basaltos formados en una dorsal oceánica o cuenca de backarc, los cuales se presentan junto con rocas sedimentarias marinas y con rocas volcánicas de arco de composición andesítica con texturas porfídicas y los basaltos (diabasas) de la unidad Diabasas de San José de Urama han sido relacionados con rocas formadas en un ambiente de plateau oceánico.

\section{AGRADECIMIENTOS}

Se agradece al Servicio Geológico Colombiano, entidad que suministró los recursos para esta investigación, al geólogo Juan Pablo Zapata, que colaboró con la elaboración de figuras; a Divid Buchs por los comentarios y el análisis químico de muestras en la Universidad de Cardiff y a los dos correctores que con sus comentarios al manuscrito incidieron en la mejora de este trabajo.

\section{REFERENCIAS}

Blanco-Quintero, I.F., García-Casco, A., Toro, L.M., Moreno, M., Ruiz, E.C., Vinasco, C.J., Cardona, A., Lázaro, C., and Morata, D. (2014). Late Jurassic terrane collision in the northwestern margin of Gondwana (Cajamarca Complex, eastern flank of the Central Cordillera, Colombia). International Geology Review, 56(15), 1852-1872.

Echeverría, L. (1973). Zonación metamórfica del Valle de Aburrá y sus alrededores. Tesis, Universidad Nacional de Colombia, Medellín.

González, H. (2001). Mapa geológico del departamento de Antioquia, escala 1:400.000, memoria explicativa INGEOMINAS. 240p.

Hall, R., Alvarez, J., y Rico, H. (1972). Geología de la parte de los departamentos de Antioquia y Caldas (Subzona II-A). Boletín Geológico, 20(1), 1-85. INGEOMINAS. 
Irvine, T.N., and Baragar, W.R.A. (1971). A guide to the chemical classification of the common volcanic rocks. Canadian Journal of Earth Sciences, 8(5), 523-548. doi: 10.1139/e71-055.

Jensen, L. (1976). A new cation plot for classifying subalakalic volcanic rocks. Ontario Division Mines, Miscellaneous, Paper 66, 22p.

Kerr, A., Marriner, G., Tarney, J., Nivia, A., Saunders, A., Thirlwall, M., and Sinton, C. (1997). Cretaceous basaltic terranes in western Columbia: elemental chronological and $\mathrm{Sr}-\mathrm{Nd}$ isotopic constrainst on petrogenesis. Journal of Petrology, 38(6), 677-702. doi: 10.1093/petroj/38.6.677.

Leal-Mejía, H. (2011). Phanerozoic gold metallogeny in the Colombian Andes: A tectono-magmatic approach. PhD Thesis. Universitat de Barcelona, España.

Le Bas, M., Le Maitre, R., Streckeisen, A., and Zannetin, B. (1986). A chemical classification of volcanic rocks based on the Total Alkali - Silica diagram. Journal of Petrology, 27(3), 745-750. doi: 10.1093/petrology/27.3.745.

Martens, U., Restrepo, J.J, Ordoñez, O., and Correa, A. (2014). The Tahamí and Anacona terranes of the Colombian Andes: Missing links between the South American and Mexican Gondwana margins. The Journal of Geology, 122(5), 507-530.

Maya, M., y González, H. (1995). Unidades litodémicas en la Cordillera Central de Colombia. Boletín Geológico, 35(2-3), 43-57. INGEOMINAS.

Maya, M. (2001). Distribución, facies y edad de las rocas metamórficas de Colombia. Informe, INGEOMINAS, 54p.

McDonough, W.F., and Sun, S.S. (1995). The composition of the Earth. Chemical Geology, 120(3-4), 223-253. doi: 10.1016/00092541(94)00140-4.

Nakamura, N. (1974). Determination of REE, Ba, $\mathrm{Fe}, \mathrm{Mg}, \mathrm{Na}$ and $\mathrm{K}$ in carbonaceous and ordinary chondrites. Geochimica et Cosmochimica Acta, 38(5), 757-775.
Nivia, A., Marriner, G., y Kerr, A. (1996). El Complejo Quebradagrande; una posible cuenca marginal intracratónica del Cretáceo Inferior en la Cordillera Central de los Andes Colombianos. VII Congreso Colombiano de Geología. Bogotá, Colombia.

Pearce, J. (1982). Trace element characteristics of lavas from destructive plate boundaries. In: R.S. Thorpe (Ed.). Andesites: Orogenic Andesites and Related Rocks (pp. 525-548). Chichester: John Wiley and Sons.

Pearce, J., and Peate, D. (1995). Tectonic implications of the composition of volcanic arc magmas. Annual Review Earth and Planetary Science, 23, 251-85. doi: 10.1146/annurev.ea.23.050195.001343.

Pearce, J.A., Stern, R.J., Bloomer, S.H., and Fryer, P. (2005). Geochemical mapping of the Mariana arc-basin system: Implications for the nature and distribution of subduction components. Geochemistry, Geophysics, Geosystems, 6(7), 1-27. doi: 10.1029/2004GC000895.

Pearce, J.A. (2008). Geochemical fingerprinting of oceanic basalts with applications to ophiolite classification and the search for Archean oceanic crust. Lithos, 100(1-4), 14-48. doi: 10.1016/j. lithos.2007.06.016.

Pearce, J.A. (2014). Geochemical fingerprinting of the Earth's Oldest Rocks. Geology, 42(2), 175-176. doi: 10.1130/focus022014.1.

Restrepo, J.J., y Toussaint, J.F. (1984). Unidades litológicas de los alrededores de Medellín. I Conferencia de Riesgos Geológicos del Valle de Aburra. Medellín, Colombia.

Restrepo, J.J., and Toussaint, J.F. (1988). Terranes and continental accretion in the Colombian Andes. Episodes, 11(3), 189-193.

Restrepo, J.J., Ibañez-Mejía, M., and García-Casco, A. (2012). U-Pb zircon ages of the Medellin Amphibolites (Central Cordillera of Colombia) reveal mid-Cretaceous tectonic juxtaposition of Triassic and mid-Cretaceous metamorphic complexes. VIII Simposio Sudamericano de Geología Isotópica. Medellín, Colombia. 
Restrepo, J.J., Ordóñez-Carmona, O., Martens, U., y Correa, A.M. (2009). Terrenos, complejos y provincias en la Cordillera Central de Colombia. Ingeniería, Investigación y Desarrollo, 9(2), 49-56.

Restrepo, J.J., Ordóñez-Carmona, O., Armstrong, R., and Pimentel, M.M. (2011). Triassic metamorphism in the northern part of the Tahamí Terrane of the central cordillera of Colombia. Journal of South American Earth Sciences, 32(4), 497-507. doi: 10.1016/j.jsames.2011.04.009.

Rodríguez, G., y Arango, M. (2013). Formación Barroso: arco volcánico toleítico y Diabasas de San José de Urama: un prisma acrecionario T-Morb en el segmento norte de la Cordillera Occidental de Colombia. Boletín de Ciencias de la Tierra, 33, 17-38.

Rodríguez, G., Arango, M., y Bermúdez, J. (2012). Batolito de Sabanalarga, plutonismo de arco en la zona de sutura entre las cortezas oceánica y continental de los Andes del Norte. Boletín de Ciencias de la Tierra, 32, 81-98.

Rodríguez, G., y Cetina, L.M. (2016). Caracterización petrográfica y química de rocas de corteza oceánica del Complejo Quebradagrande y comparación con rocas de unidad Diabasas de San José de Urama. Boletín de Geología, 38(3), 15-29. doi: 10.18273/revbol.v38n3-2016001.

Rodríguez, G., y Correa-Martínez, A.M. (2015). Edades $\mathrm{U}-\mathrm{Pb}$ en circón de varias unidades metamórficas al este y noreste de la ciudad de Medellín, Cordillera Central de Colombia. XV Congreso Colombiano de Geología, Bucaramanga, Colombia, pp. 287289.

Rodríguez, G., González, H., Zapata, G., Cossio, U., y Correa-Martínez, A.M. (2016). Geología de la plancha 147 Medellín oriental, escala 1:50.000 versión 2016. 464p.

Rodríguez, G., Obando, G., Correa-Martínez, A.M., Zapata, G., Correa, T., Obando, M., Rincón, A., y
Zapata, J.P. (2017). Redefinición del bloque norte del Batolito de Ibagué con base en nuevos datos de petrografía, litogeoquímica y geocronología U-Pb. XVI Congreso Colombiano de Geología, Santa Marta, Colombia, pp. 1443-1447.

Rodríguez, G., y Zapata, G. (2013). Análisis comparativo entre la Formación Barroso y el Complejo Quebradagrande: un arco volcánico toleítico-calcoalcalino, segmentado por el sistema de fallas de Romeral en los Andes del Norte?. Boletín de Ciencias de la Tierra, 33, 39-58.

Sun, S.S., and McDonough, W.F. (1989). Chemical and isotopic systematics of oceanic basalts: implications for mantle composition and processes. In: A.D. Sanders, and M.J. Norry (Eds.). Magmatism in the ocean basins (pp. 313-345). Vol. 42. London: Geological Society, Special Publications.

Vinasco, C., Cordani, U.G., González, H., Weber, M., and Peláez, C. (2006). Geochronological, isotopic, and geochemical data from Permo-Triassic granitic gneisses and granitoids of the Colombian Central Andes. Journal of South American Earth Sciences, 21(4), 355-371.

Wilson, B.M. (1989). Igneous petrogenesis. A global tectonic approach. London: Unwin Hyman.

Winter, J.D. (2001). An introduction to igneous and metamorphic petrology. New Jersey: Prentice Hall.

Gabriel Rodríguez-García ORCID: 0000-0003-1422-3523

Carlos Mario Celada-Arango ORCID: 0000-0002-3263-3764

Trabajo recibido: febrero 23 de 2018 Trabajo aceptado: mayo 23 de 2018 